\title{
The impact of blood flow rate during hemodialysis on all-cause mortality
}

Kyung Yoon Chang ${ }^{1}$, Su-Hyun Kim² ${ }^{2}$, Young Ok Kim ${ }^{1}$, Dong Chan Jin ${ }^{1}$, Ho Chul Song ${ }^{1}$, Euy Jin Choi ${ }^{1}$, Yong-Lim Kim ${ }^{3}$, Yon-Su Kim ${ }^{4}$, Shin-Wook Kang ${ }^{5}$, Nam-Ho Kim ${ }^{6}$, Chul Woo Yang ${ }^{1}$, and Yong Kyun Kim ${ }^{1,7}$

${ }^{1}$ Department of Internal Medicine, College of Medicine, The Catholic University of Korea, Seoul; ${ }^{2}$ Department of Internal Medicine, Chung-Ang University College of Medicine, Seoul; ${ }^{3}$ Department of Internal Medicine, Kyungpook National University School of Medicine, Daegu; ${ }^{4}$ Department of Internal Medicine, Seoul National University College of Medicine, Seoul; ${ }^{5}$ Department of Internal Medicine, Yonsei University College of Medicine, Seoul; ${ }^{6}$ Department of Internal Medicine, Chonnam National University Medical School, Gwangju; ${ }^{7}$ Cell Death Disease Research Center, College of Medicine, The Catholic University of Korea, Seoul, Korea

Received: April 22, 2015

Revised : July 4, 2015

Accepted: July 8, 2015

\section{Correspondence to}

\section{Yong Kyun Kim, M.D.}

Department of Internal Medicine, College of Medicine, Bucheon St.

Mary's Hospital, The Catholic

University of Korea, 327 Sosa-ro,

Wonmi-gu, Bucheon 14647, Korea

Tel: +82-32-340-7019

Fax: +82-32-340-2667

E-mail:drkimyk@catholic.ac.kr
Background/Aims: Inadequacy of dialysis is associated with morbidity and mortality in chronic hemodialysis (HD) patients. Blood flow rate (BFR) during HD is one of the important determinants of increasing dialysis dose. However, the optimal BFR is unclear. In this study, we investigated the impact of the BFR on allcause mortality in chronic HD patients.

Methods: Prevalent HD patients were selected from Clinical Research Center registry for end-stage renal disease cohort in Korea. We categorized patients into two groups by $\mathrm{BFR}<250$ and $\geq 250 \mathrm{~mL} / \mathrm{min}$ according to the median value of BFR 250 $\mathrm{mL} / \mathrm{min}$ in this study. The primary outcome was all-cause mortality.

Results: A total of 1,129 prevalent HD patients were included. The number of patients in the $\mathrm{BFR}<250 \mathrm{~mL} / \mathrm{min}$ was $271(24 \%)$ and in the $\mathrm{BFR} \geq 250 \mathrm{~mL} / \mathrm{min}$ was 858 (76\%). The median follow-up period was 30 months. Kaplan-Meier analysis showed that the mortality rate was significantly higher in patients with $\mathrm{BFR}<250$ $\mathrm{mL} / \mathrm{min}$ than those with BFR $\geq 250 \mathrm{~mL} / \mathrm{min}$ ( $p=0.042$, log-rank). In the multivariate Cox regression analyses, patients with $\mathrm{BFR}<250 \mathrm{~mL} / \mathrm{min}$ had higher allcause mortality than those with $\mathrm{BFR} \geq 250 \mathrm{~mL} / \mathrm{min}$ (hazard ratio, 1.66; $95 \%$ confidence interval, 1.00 to $2.73 ; p=0.048$ ).

Conclusions: Our data showed that BFR $<250 \mathrm{~mL} / \mathrm{min}$ during HD was associated with higher all-cause mortality in chronic HD patients.

Keywords: Mortality; Renal dialysis; Blood flow rate; Dialysis adequacy

\section{INTRODUCTION}

Adequate dialysis is critically important in reducing morbidity and mortality in maintenance hemodialysis (HD) patients [1]. Kt/V is the commonly used marker for dialysis adequacy [1]. Kt/V can be modified by efficiency of dialyzer, duration of dialysis, dialysis frequency, dialysate flow rate and dialyzer blood flow rate (BFR) [2-4]. BFR is one of the most important determinants for achieving of adequate $\mathrm{Kt} / \mathrm{V}$ [2]. In general, increasing dialyzer BFR increase in delivered Kt/V [2,5]. A previous study reported that a $30 \%$ increase in $\mathrm{BFR}$, giving the 
same surface, membrane and dialysate flow, resulted in a $23 \%$ increase in urea clearance [5]. Therefore, it may be postulated that lower BFR may cause inadequate dialysis and influence on clinical outcomes in HD patients. However, there are few studies on the association between BFR and mortality in HD patients and the optimal BFR is unclear.

Japan Dialysis Outcomes and Practice Patterns Study (DOPPS) reported that patients with a mean prescribed $\mathrm{BFR}<180 \mathrm{~mL} / \mathrm{min}$ had higher mortality compared with reference group of BFR 180 to $210 \mathrm{~mL} / \mathrm{min}$ [6]. However, it is uncertain whether these results can be generalized to other country groups because the prescribed BFRs in Japanese HD patients is usually $200 \mathrm{~mL} / \mathrm{min}$, which are lower than those in other countries with 250 to $350 \mathrm{~mL} /$ min $[6,7]$. Furthermore, some investigators proposed that the use of low BFR in Japan may contribute the longer survival compared other countries because the lower BFR may help facilitate the maintenance of high rate of arteriovenous fistula (AVF) $[6,8]$.

In this study, we investigated the impact of the BFR on all-cause mortality in chronic HD patients from the Clinical Research Center (CRC) registry for end-stage renal disease (ESRD), a multicenter prospective observational study in Korea.

\section{METHODS}

\section{Study population}

All patients in this study participated in the CRC registry for ESRD. The cohort started in April 2009 and included adult ( $>18$ years of age) dialysis patients. A total of 1,669 prevalent HD patients were enrolled. We excluded patients whose BFR could not be ascertained at enrollment $(n=4)$, duration of HD therapy was less than 1 year $(\mathrm{n}=392)$, session of HD was not three times per week ( $\mathrm{n}$ $=141$ ), treatment time per HD session was more than 5 hours $(n=4)$, or dialysis modality was hemodiafiltration $(n=57)$. So, 1,129 patients were included in the final analysis.

Demographic data and clinical data were collected at enrollment. Assessment of dialysis characteristics and measurements of health were performed every 6 months until the end of follow-up. Dates and causes of mortality were immediately reported through the follow-up pe- riod. The CRC registry for ESRD was approved by the medical ethics committees of all of the participating hospitals and informed consent was obtained from all patients before inclusion.

Baseline demographic data and clinical data including age, gender, height, weight, body mass index (BMI), systolic blood pressure (SBP), diastolic blood pressure (DBP), causes of ESRD, comorbidities, laboratory investigations, and therapeutic characteristics were recorded. Serum hemoglobin levels, creatinine levels, total cholesterol (TC) levels, albumin levels, calcium levels, phosphorus levels, intact parathyroid hormone (iPTH) levels, and $\beta 2$-microglobulinlevels were determined from blood samples. The single-pool Kt/V (spKt/V) was determined by two-point urea modeling based on the intradialytic reduction in blood urea and intradialytic weight loss.

BFR at study enrollment was based on prescribed value. In this study, the median value of BFR was $250 \mathrm{~mL} /$ $\mathrm{min}$. We categorized patients into two groups by BFR < $250 \mathrm{~mL} / \mathrm{min}$ and $\mathrm{BFR} \geq 250 \mathrm{~mL} / \mathrm{min}$ according to median value of $B F R$.

\section{Outcomes}

The clinical outcome of this study was all-cause mortality. The primary outcome was all-cause mortality. All patients were followed until death (event) or the end of the study, with censoring of data at the time a patient underwent renal transplantation or was lost to followup because of patient's refusal of further participation or patient's transfer to a nonparticipating hospital. For each death, the clinical center principal investigator completed a form that included cause of death according to the CRC for ESRD study classification.

\section{Statistical analysis}

Data with continuous variables and normal distribution are presented as mean $\pm \mathrm{SD}$ and those without normal distribution are presented as the median with ranges as appropriate for the type of variable. The Student $t$ test and Mann-Whitney test were used, as appropriate, to determine the differences in continuous variables. Categorical variables are presented as percentages. The Pearson chi-square test or Fisher exact test was used to determine the differences in categorical variables.

Absolute mortality rates were calculated per 100 person-years of follow-up. The survival curves were esti- 
mated by the Kaplan-Meier method and compared by the log-rank test between the group with BFR $<250$ and $\geq 250 \mathrm{~mL} / \mathrm{min}$ group. The Cox proportional hazard regression model was used to calculate the hazard ratio (HR) with 95\% confidence interval (CI) for all-cause mortality. The assumption of proportional hazards over time was assessed by visual inspection of a log-minuslog survival plot. The Cox models were adjusted for significant or nearly significant $(p<0.1)$ predictor for all-cause mortality in univariate Cox regression analysis including age, sex, diabetes mellitus, coronary artery disease, types of dialyzer membrane, serum hemoglobin, serum albumin, and serum TC. To have adequate confounder control, important covariates known to be influential based on prior studies and clinical insight, such as SBP, and spKt/V were retained in the multivariate Cox regression model regardless of their statistical significance. A value of $p<0.05$ was considered statistically significant. All statistical analyses were performed using SPSS version 16.0 (SPSS Inc., Chicago, IL, USA).

\section{RESULTS}

\section{Patient characteristics}

A total of 1,129 prevalent HD patients were included in the present analysis. The mean age of patients was $58 \pm$ 13 years. The main causes of ESRD were diabetes (43\%), renal vascular disease (19\%), and glomerulonephritis (14\%). Fig. 1 shows the distribution of patients according to BFR. The median BFR was $250 \mathrm{~mL} / \mathrm{min}$. Baseline characteristics of the study population according to the median value of BFR are shown in Table 1. The number of patients in the BFR $<250 \mathrm{~mL} / \mathrm{min}$ was $271(24 \%)$ and in the BFR $\geq 250 \mathrm{~mL} / \mathrm{min}$ was 858 (76\%). The mean BFR of patients with the BFR $<250 \mathrm{~mL} / \mathrm{min}$ was $218 \pm 17 \mathrm{~mL} /$ min and the mean BFR of patients with the BFR $\geq 250$ $\mathrm{mL} / \mathrm{min}$ was $272 \pm 24 \mathrm{~mL} / \mathrm{min}$. Male was more prevalent in patients with the $\mathrm{BFR} \geq 250 \mathrm{~mL} / \mathrm{min}$. Diabetes was more common in patients with the $\mathrm{BFR}<250 \mathrm{~mL} / \mathrm{min}$. Patients of the $\mathrm{BFR}<250 \mathrm{~mL} / \mathrm{min}$ had a lower percentage of use of arteriovenous graft (AVG) and a higher percentage of use of catheter. Dialysate flow rate was lower in patients with the BFR $<250 \mathrm{~mL} / \mathrm{min}$. Patients of the $\mathrm{BFR}<250 \mathrm{~mL} / \mathrm{min}$ had a higher percentage of use of low-flux dialyzer membrane and a lower percentage of

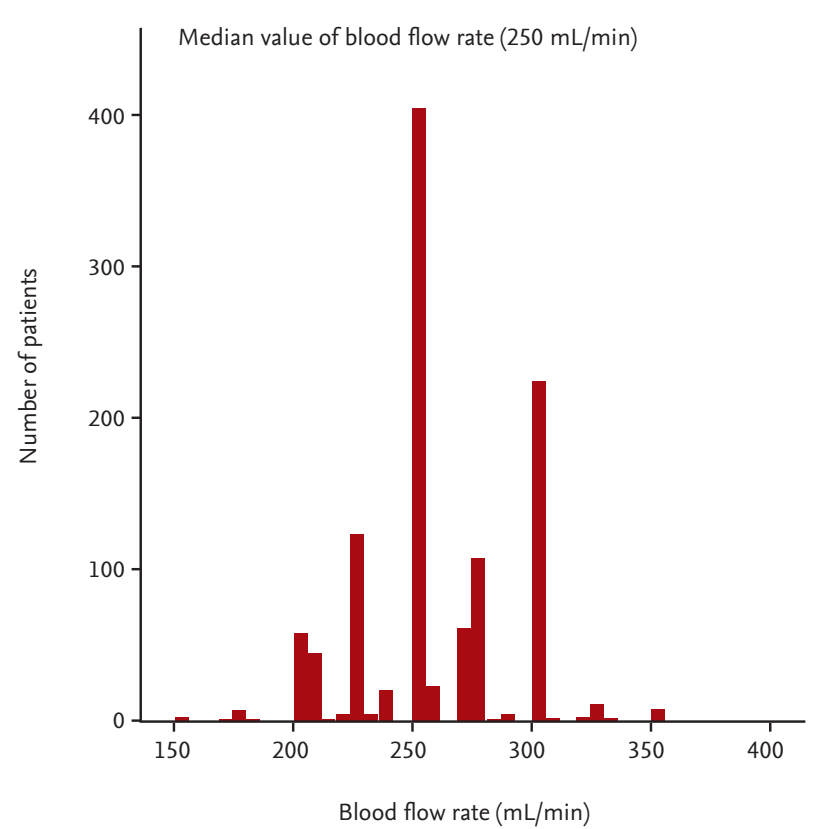

Figure 1. The distribution of enrolled patients according to blood flow rate.

use of high-flux dialyzer membrane. Serum calcium levels were lower and serum $\beta 2$-microglobulin levels were higher in patients with the BFR $<250 \mathrm{~mL} / \mathrm{min}$. SpKt $/ \mathrm{V}$ was lower in patients with the $\mathrm{BFR}<250 \mathrm{~mL} / \mathrm{min}$. There was no significant difference in age, duration of dialysis, use of AVF, treatment time of dialysis, ultrafiltration volume per post-dialysis weight, BMI, SBP, DBP, hemoglobin levels, serum TC levels, serum phosphorus levels or serum iPTH levels in each group.

\section{The impact of BFR on all-cause mortality}

The median follow-up period was 30 months (interquartile range, 18 to 41 ). During the follow-up period, 178 patients left the study. The reasons for censoring included transfer to a nonparticipating hospital $(n=65)$, kidney transplantation $(\mathrm{n}=58)$, refusal of further participation $(n=27)$, or others $(n=18)$. There were 96 deaths during the follow-up period. The leading causes of death in whole cohort were cardiovascular diseases $(35 \%$ of all deaths) and infectious diseases (27\% of all deaths). Table 2 shows the causes of deaths in each group. There was no significant difference in the causes of death between patients with the BFR $<250 \mathrm{~mL} / \mathrm{min}$ and patients with the $\mathrm{BFR} \geq 250 \mathrm{~mL} / \mathrm{min}(p=0.531)$. The absolute mortality rate was 3.53 deaths per 100 person-years. Fig. 2 shows 
Table 1. Baseline characteristics of the study population according to blood flow rate

\begin{tabular}{|c|c|c|c|}
\hline \multirow{2}{*}{ Characteristic } & \multicolumn{2}{|c|}{ Blood flow rate, $\mathrm{mL} / \mathrm{min}$} & \multirow{2}{*}{$p$ value } \\
\hline & $<250(\mathrm{n}=271)$ & $\geq 250(n=858)$ & \\
\hline Age, yr & $58 \pm 14$ & $58 \pm 13$ & 0.682 \\
\hline Male sex & $122(45.0)$ & $503(58.6)$ & $<0.001$ \\
\hline Duration of dialysis, mon & $63 \pm 58$ & $68 \pm 56$ & 0.243 \\
\hline Causes of ESRD & & & 0.033 \\
\hline Diabetes mellitus & $137(50.6)$ & $351(40.9)$ & \\
\hline Renal vascular disease & $41(15 \cdot 1)$ & $177(20.6)$ & \\
\hline Glomerulonephritis & $34(12.5)$ & $127(14.8)$ & \\
\hline Others/unknown & $59(21.8)$ & $203(23.7)$ & \\
\hline \multicolumn{4}{|l|}{ Comorbidities } \\
\hline Diabetes mellitus & $151(55 \cdot 9)$ & $407(47.8)$ & 0.020 \\
\hline Coronary artery disease & $86(31.9)$ & $298(34.9)$ & 0.364 \\
\hline \multicolumn{4}{|l|}{ Types of vascular access } \\
\hline Arteriovenous fistula & $220(81.5)$ & $658(78.1)$ & 0.242 \\
\hline Arteriovenous graft & $37(13.7)$ & $165(19.6)$ & 0.029 \\
\hline Catheter & $13(4.8)$ & $19(2.3)$ & 0.029 \\
\hline Blood flow rate, $\mathrm{mL} / \mathrm{min}$ & $218 \pm 17$ & $272 \pm 24$ & $<0.001$ \\
\hline Treatment time of dialysis, hr/wk & $12.0 \pm 0.4$ & $12.0 \pm 0.3$ & 0.747 \\
\hline UF volume per post-dialysis weight, \% & $4.0 \pm 1.8$ & $4.1 \pm 1.8$ & 0.969 \\
\hline Dialysate flow rate, $\mathrm{mL} / \mathrm{min}$ & $504 \pm 26$ & $529 \pm 71$ & $<0.001$ \\
\hline \multicolumn{4}{|l|}{ Dialyzer membrane } \\
\hline Low-flux dialyzer membrane & $161(59.9)$ & $397(46.9)$ & $<0.001$ \\
\hline High-flux dialyzer membrane & $108(40.1)$ & $450(53.1)$ & $<0.001$ \\
\hline Body mass index, $\mathrm{kg} / \mathrm{m}^{2}$ & $21.8 \pm 3.2$ & $22.2 \pm 3.3$ & 0.055 \\
\hline Systolic blood pressure, $\mathrm{mmHg}$ & $143 \pm 21$ & $142 \pm 20$ & 0.515 \\
\hline Diastolic blood pressure, $\mathrm{mmHg}$ & $77 \pm 12$ & $78 \pm 13$ & 0.587 \\
\hline Hemoglobin, g/dL & $10.6 \pm 1.3$ & $10.6 \pm 1.2$ & 0.639 \\
\hline Serum albumin, g/dL & $4.0 \pm 0.4$ & $3.9 \pm 0.4$ & 0.166 \\
\hline Serum TC, mg/dL & $154 \pm 35$ & $151 \pm 35$ & 0.217 \\
\hline Serum calcium, mg/dL & $8.9 \pm 0.8$ & $9.1 \pm 0.8$ & 0.012 \\
\hline Serum phosphorus, mg/dL & $5.0 \pm 1.7$ & $4.9 \pm 1.5$ & 0.265 \\
\hline Serum iPTH, pg/mL & $146.5(56.0-295.0)$ & $115(51.1-249 \cdot 5)$ & 0.137 \\
\hline Serum $\beta 2$-microglobulin, mg/L & $36.6(24.7-47.4)$ & $26.8(22.3-34.2)$ & $<0.001$ \\
\hline $\mathrm{spKt} / \mathrm{V}$ & $1.44 \pm 0.29$ & $1.49 \pm 0.30$ & 0.017 \\
\hline
\end{tabular}

Values are presented as mean $\pm \mathrm{SD}$, number (\%), or median (interquartile range).

ESRD, end-stage renal disease; UF, ultrafiltration; TC, total cholesterol; iPTH, intact parathyroid hormone; spKt/V, single-pool $\mathrm{Kt} / \mathrm{V}$.

the Kaplan-Meier plot of patient survival according to BFR. Kaplan-Meier analysis showed that the mortality rate was significantly higher in patients with $\mathrm{BFR}<250$ $\mathrm{mL} / \mathrm{min}$ than those with $\mathrm{BFR} \geq 250 \mathrm{~mL} / \mathrm{min}(p=0.042$, log-rank).
Univariate and multivariate Cox regression analysis for all-cause mortality are shown in Table 3. In crude model, the HR for all-cause mortality of BFR $<250 \mathrm{~mL} /$ min was 1.56 (95\% CI, 1.01 to $2.40 ; p=0.044$ ). In multivariate Cox regression analysis, patients with $\mathrm{BFR}<250 \mathrm{~mL} /$ 
Table 2. Causes of deaths in each group

\begin{tabular}{lcc}
\hline Disease & \multicolumn{2}{c}{ Blood flow rate, $\mathrm{mL} / \mathrm{min}$} \\
\cline { 2 - 3 } & $<250(\mathrm{n}=271)$ & $\geq 250(\mathrm{n}=858)$ \\
\hline Cardiovascular diseases including cerebrovascular diseases, $\mathrm{n}$ (\% of deaths) & $9(30.0)$ & $25(37.9)$ \\
Infectious diseases, n (\% of deaths) & $11(36.7)$ & $15(22.7)$ \\
Malignancy, n (\% of deaths) & $1(3.3)$ & $4(6.1)$ \\
Others, n (\% of deaths) & $9(30.0)$ & $22(33.3)$ \\
Total, n (\% of each group) & $30(11.1)$ & $66(7.7)$ \\
\hline
\end{tabular}

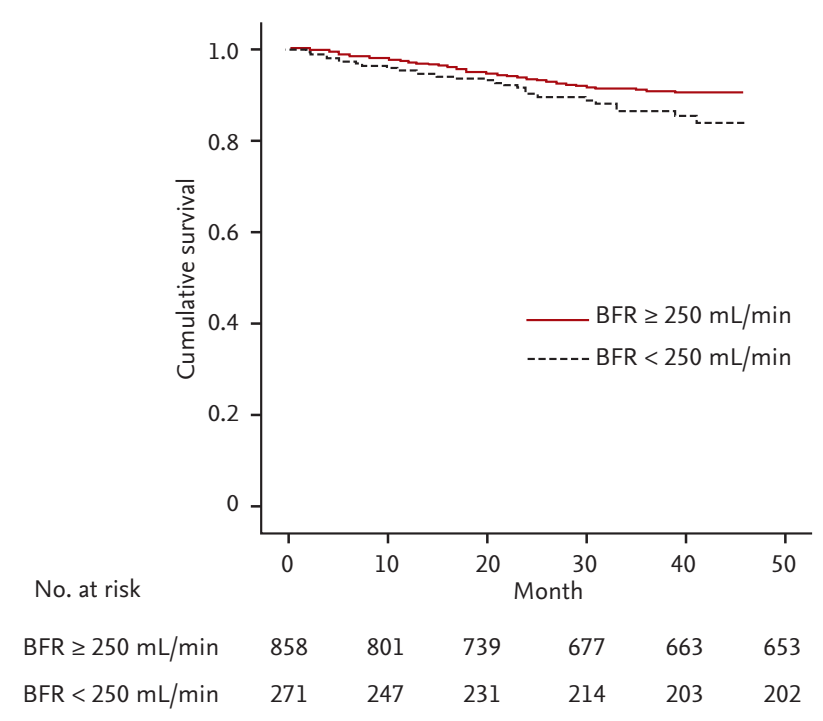

Figure 2. Kaplan-Meier survival curve for all-cause mortality $(p=0.042$ by log-rank test).

min had a significantly higher risk for all-cause mortality than those with $B F R \geq 250 \mathrm{~mL} / \mathrm{min}$ even after adjusting for demographics, laboratory data, and comorbid conditions (model 1: HR, 1.60; 95\% CI, 1.03 to 2.47 ; $p=$ 0.036; model 2: HR, 1.66; 95\% CI, 1.00 to $2.73 ; p=0.048$ ).

\section{Subgroup analyses according to baseline covariates}

To determine the interaction between baseline covariates and BFR for all-cause mortality, we divided the entire study population into subgroups according to various baseline covariates and performed subgroup analyses. We found that age, spKt/V, gender, diabetes, types of vascular access, and types of dialyzer membrane significantly interacted with BFR for mortality. As shown in Fig. 3, all-cause mortality rate was significantly higher in patients with $\mathrm{BFR}<250 \mathrm{~mL} / \mathrm{min}$ than those with $\mathrm{BFR} \geq 250 \mathrm{~mL} / \mathrm{min}$ in subgroup with older age group (HR, 1.96; 95\% CI, 1.13 to 3.39; $p=0.017$ ), below median values of spKt/V 1.5 (HR, 3.01; 95\% CI, 1.55 to $5.86 ; p=0.001)$, male (HR, 1.83; $95 \% \mathrm{CI}, 1.03$ to $3.28 ; p=$ 0.041), diabetes (HR, 2.07; 95\% CI, 1.19 to $3.58 ; p=0.010$ ), $\operatorname{AVF}(\mathrm{HR}, 2.09$; 95\% CI, 1.24 to 3.53; $p=0.006$ ), and lowflux dialyzer membrane (HR, 2.06; $95 \%$ CI, 1.20 to 3.52; $p=0.009)$, whereas there was no significant difference in all-cause mortality between two BFR groups in subgroup with younger age group (HR, o.89; 95\% CI, 0.36 to 2.19; $p=0.797)$, above median values of spKt/V $1.5(\mathrm{HR}$, $0.72 ; 95 \%$ CI, 0.28 to $1.88 ; p=0.501$ ), female (HR, 1.45; $95 \%$ $\mathrm{CI}, 0.71$ to $2.97 ; p=0.313$ ), non-diabetes (HR, 0.90; 95\% CI, 0.38 to $2.13 ; p=0.807), \mathrm{AVG}(\mathrm{HR}, 0.67 ; 95 \% \mathrm{CI}, 0.19$ to 2.37 ; $p=0.536)$, and high-flux dialyzer membrane (HR, 0.71 ; $95 \% \mathrm{CI}, 0.24$ to $2.06 ; p=0.524$ ).

\section{DISCUSSION}

In this multicenter prospective observational study to investigate the impact of BFR on mortality in chronic HD patients, we demonstrated that patients with BFR < $250 \mathrm{~mL} / \mathrm{min}$ had higher risk for all-cause mortality.

The BFR is different in many countries and the optimal BFR has been unclear. The DOPPS has shown that in the United States, the patients that BFR is more than $400 \mathrm{~mL} / \mathrm{min}$ account for $83.6 \%$ of the HD patients [9]. In Canada and other Europe, the patients with BFR $\geq 250$ $\mathrm{mL} / \mathrm{min}$ take about $98 \%$ of the HD patients [9]. However, in Japan, the patients with BFR $\geq 250 \mathrm{~mL} / \mathrm{min}$ account for $18 \%$ and BFR prescribed usually $200 \mathrm{~mL} / \mathrm{min}$ for a typical HD treatment $[6,9]$.

The impact of BFR on survival has been controversial. Some investigators proposed that the use of low BFR in 
Table 3. The univariate and multivariate Cox regression analysis for all-cause mortality

\begin{tabular}{|c|c|c|c|c|c|c|c|c|c|}
\hline & \multicolumn{3}{|c|}{ Crude model } & \multicolumn{3}{|c|}{ Model $1^{\mathrm{a}}$} & \multicolumn{3}{|c|}{ Model $2^{b}$} \\
\hline & HR & $95 \% \mathrm{CI}$ & $p$ value & HR & $95 \% \mathrm{CI}$ & $p$ value & HR & $95 \% \mathrm{CI}$ & $p$ value \\
\hline $\mathrm{BFR}<250 \mathrm{~mL} / \mathrm{min}($ vs. $\geq 250)$ & 1.56 & $1.01-2.40$ & 0.044 & 1.60 & $1.03-2.47$ & 0.036 & 1.66 & $1.00-2.73$ & 0.048 \\
\hline
\end{tabular}

HR, hazard ratio; CI, confidence interval; BFR, blood flow rate.

${ }^{a}$ Model 1: multivariate model including age and sex.

${ }^{\mathrm{b}}$ Model 2: multivariate model including model 1 + diabetes mellitus, coronary artery disease, systolic blood pressure, serum hemoglobin, serum albumin, and serum total cholesterol, types of dialyzer membrane, and single-pool Kt/V.

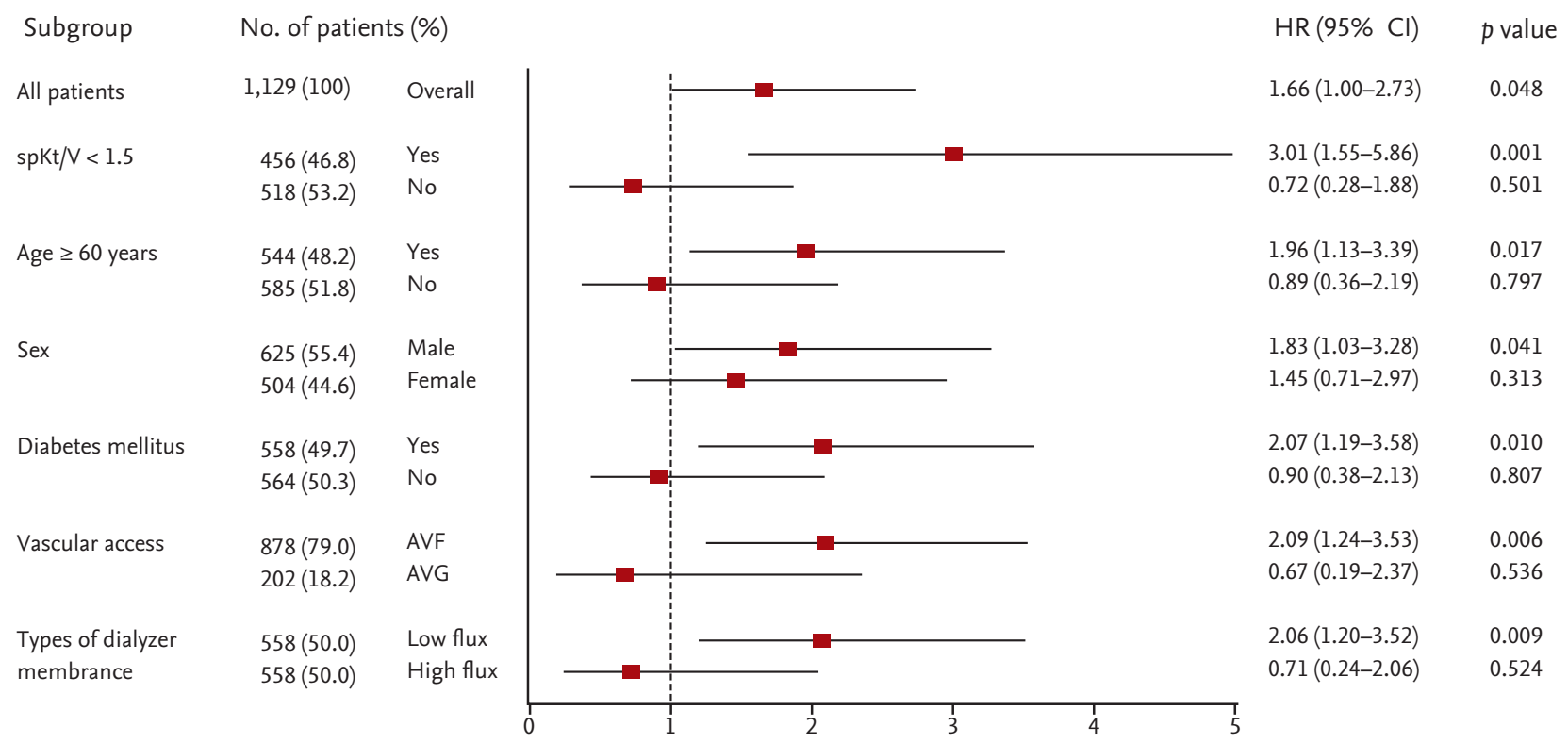

Figure 3. Subgroup analyses for all-cause mortality of patients with blood flow rate $<250 \mathrm{~mL} / \mathrm{min}$ according to age, singlepool Kt/V (spKt/V), gender, diabetes, types of vascular access, and types of dialyzer membrane. HR, hazard ratio; CI, confidence interval; AVF, arteriovenous fistula; AVG, arteriovenous graft.

Japan may contribute the longer survival compared other countries because the lower BFR may help facilitate the maintenance of high rate of AVF. Some investigators suggest that the use of low BFR may contribute the longer survival by facilitating the maintenance of high rate of AVF $[6,8]$. On the contrary, other studies demonstrated that increased BFR is important for optimizing dialysis dose $[2,5]$ and inadequate dialysis dose is associated with increased mortality [1].

In this study, the mean BFR was $259 \pm 32 \mathrm{~mL} / \mathrm{min}$ and the patients with $B F R \geq 250 \mathrm{~mL} / \mathrm{min}$ accounts for $76 \%$ in the whole cohort, of which was higher than that of Japan and lower than that of the United States. Our findings, which showed that lower BFR was associated with higher all-cause mortality rate, provide the evidence for the association between BFR and all-cause mortality.

The mechanisms underlying the risk of all-cause mortality associated with lower BFR are unclear. Inadequate doses of dialysis promote atherosclerosis, infection, and malnutrition, which in turn will increase mortality [10].

Does lower BFR contribute to the inadequate dialysis dose? Previous studies have shown the relationship between BFR and dialysis dose. Borzou et al. [11] reported that $16.7 \%$ of patients had Kt/V higher than 1.3 using BFR of $200 \mathrm{~mL} / \mathrm{min}$, while $26.2 \%$ of patients had Kt/V higher than 1.3 using BFR of $250 \mathrm{~mL} / \mathrm{min} \mathrm{HD}$. Kim et al. [12] reported that by increasing the BFR by $15 \%$ to $20 \%$ in patients with low efficiency dialysis (Kt/V less than of 1.2), efficiency of dialysis would increase $4 \%$. Similar with previous studies, BFR was positively correlated 
with spKt/V in this study $(\beta=0.108, p=0.001)$. Odds ratio of patients with $\mathrm{BFR}<250 \mathrm{~mL} / \mathrm{min}$ to have inadequate dialysis dose (spKt/V $\leq 1.2$ ) was 1.5 (95\% CI, 1.03 to 2.20; $p$ $=0.036)$ in this study. These findings suggest that lower BFR is associated with inadequate dialysis.

Next, does increased spKt/V by increasing BFR influence on mortality? The Hemodialysis (HEMO) Study study has demonstrated that increasing spKt/V above standard dose did not impact survival [13]. Therefore, it may be possible to postulate that, if BFR is associated with delivered dialysis dose, the impact of BFR on mortality may be more prominent in patients with lower dialysis dose rather than patients with higher dialysis dose. Our data support this postulation. As shown in Fig. 3, in subgroup analyses according to median value of spKt/V 1.5, BFR < $250 \mathrm{~mL} / \mathrm{min}$ was significantly associated with all-cause mortality in subgroup with below median value of spKt/V, but not in subgroup with above median spKt/V. We cautiously suggest that lower BFR contributed inadequate dialysis, which may be associated with higher mortality especially in HD patients with lower dialysis dose. Randomized controlled trial may elucidate the association between BFR and clinical outcomes.

The dialysis treatment time is a main determinant of $\mathrm{Kt} / \mathrm{V}$. In this study, the dialysis treatment time for the two BFR groups was comparable in spite of the presence of significant difference in spKt/V. It may be due that most patients (96\%) have same dialysis time, $12 \mathrm{hr} / \mathrm{wk}$. Similar dialysis treatment time between two BFR groups may reduce the interaction of treatment time with $\mathrm{Kt} / \mathrm{V}$ and mortality, which may contribute to clarify the association of BFR with Kt/V and mortality in this study.

What would happen to the outcome if patients with low BFR received dialysis with extended dialysis treatment time? Japan DDOPS study reported that low Kt/V was more strongly associated with short dialysis treatment time than low BFR [6]. Short dialysis treatment time is associated with low $\mathrm{Kt} / \mathrm{V}$ and independent risk factor for sudden death $[3,6]$. Furthermore, DOPPS showed longer HD patients survival with longer dialysis treatment time [7]. Extend dialysis treatment time in lower BFR group may increase Kt/V or overcome dialysis inadequacy, which in turn reduce all-cause mortality. Therefore, the impact of lower BFR on all-cause mortality should be cautiously interpreted in consideration that HD patients may have different dialysis treatment time according to the dialysis centers.

Another explanation for the association between lower BFR and higher all-cause mortality may be possible. Gutzwiller et al. [14] reported that increasing BFR was effective in clearance of potassium. It may be postulated that higher BFR may contribute correction of electrolyte imbalance in HD patients, which in turn decrease cardiovascular mortality from electrolyte imbalance such as hyperkalemia.

One of the interesting findings of this study is that spKt/V had no significant association with all-cause mortality. Kaplan-Meier analysis showed that the mortality rate was not significantly different between patients with spKt $/ \mathrm{V}<1.5$ (the median value of $\mathrm{spKt} / \mathrm{V}$ in this study) and those with spKt $/ \mathrm{V} \geq 1.5$ ( $p=0.680, \log$ rank). Cox regression analyses showed that there was no significant difference in all-cause mortality between patients with spKt/V $<1.5$ and those with spKt/V $\geq 1.5$ (HR, 0.85 ; $95 \%$ CI, 0.53 to $1.38 ; p=0.515)$. The thresholder of $\mathrm{Kt} / \mathrm{V}$ used in clinical practice is 1.2. When the patients were divided according to $\mathrm{Kt} / \mathrm{V}$ 1.2, similar data were obtained (data not shown). These findings are consistent with the recent notion of Kt/V [15]. Although Kt/V is a useful marker of dialysis adequacy, clinical studies have shown that Kt/V is not often the sole and the most consistent determinant of dialysis outcomes [13]. It may be due that $\mathrm{Kt} / \mathrm{V}$ cannot properly cover up for diverse recent dialysis strategic alternatives, such as extended or daily dialysis, high-flux dialysis, and convective strategies [15]. Unlike spKt/V, BFR was independent risk factor for all-cause mortality in this study. Furthermore, BFR had significant association with all-cause mortality after adjustment for spKt/V (Table 3). These findings suggest that lower BFR may have independent prognostic value on all-cause mortality besides reflecting dialysis inadequacy.

Another interesting finding of our study is that age, gender, diabetes, types of vascular access, and types of dialyzer membrane significantly interacted with BFR for all-cause mortality. These findings suggest that lower BFR was associated with higher all-cause mortality especially in subgroup with older age, male, diabetes, use of AVF, and use of low-flux dialyzer membrane. The reasons for interaction of these factors with BFR-related mortality are unclear. It may be cautiously postulated 
that the survival benefit of the optimizing dialysis adequacy by higher BFR may be more prominent in patients with older age, male, diabetes, use of AVF, and use of low-flux dialyzer membrane. To clarify the precise mechanism, further studies are needed.

Our study has some potential limitations. First, our study was an observational study and patients were not randomly allocated to the two groups. Therefore, this study may have been limited by selection bias. Despite careful adjustment for clinical variables, unknown confounding variables may not have been adequately adjusted for in the multivariable analyses. Second, in spite of the multicenter nature of the study, the cohort consisted of Korean patients and all were Asian. Thus, it is uncertain whether our results can be generalized to other ethnic groups. Third, the causes of low BFR such as vascular access dysfunction and hemodynamic instability were not assessed in this cohort study. Forth, in spite of dividing the patients according to median value of BFR $250 \mathrm{~mL} / \mathrm{min}$, there is huge difference in number of patients between two groups. It may be due to the discrete and skewed distribution of BFR. As shown in Fig. 1, because substantial number of patients (404 patients of the enrolled patients) had median value of BFR ( $250 \mathrm{~mL} /$ $\mathrm{min})$, the number of patients with $\mathrm{BFR} \geq 250 \mathrm{~mL} / \mathrm{min}$ was greater than the number of patients with $\mathrm{BFR}<250$ $\mathrm{mL} / \mathrm{min}(\mathrm{n}=858$ vs. $\mathrm{n}=271$, respectively).

In conclusion, the patients with BFR $<250 \mathrm{~mL} / \mathrm{min}$ is independently associated with increased all-cause mortality, especially in patients with older age, spKt/V $<1.5$, male, diabetes, use of AVF and use of low-flux dialyzer membrane. Our results suggest that monitoring and careful attention of the factors decreasing BFR may be helpful to improve clinical outcomes in chronic HD patient.

\section{KEY MESSAGE}

1. The blood flow rate $(\mathrm{BFR})<250 \mathrm{~mL} / \mathrm{min}$ during hemodialysis (HD) was associated with increased all-cause mortality in chronic HD patients.

2. Our results suggest that monitoring and careful attention of the factors decreasing BFR may be helpful to improve clinical outcomes.

\section{Conflict of interest}

No potential conflict of interest relevant to this article was reported.

\section{Acknowledgments}

This work was supported by a grant of the Korea Healthcare Technology R\&D Project, Ministry of Health and Welfare, Republic of Korea (HI10C2O2O). We thank the study coordinators Hye Young Lim, Nam Hee Kim, Mi Joung Moon, Hwa Young Lee, Mi Joung Kwon, Su Yeon An, Su Joung Oh, and Hye Young Kwak for contribution to this study.

\section{REFERENCES}

1. Held PJ, Port FK, Wolfe RA, et al. The dose of hemodialysis and patient mortality. Kidney Int 1996;50:550-556.

2. Mandolfo S, Borlandelli S, Ravani P, Imbasciati E. How to improve dialysis adequacy in patients with vascular access problems. J Vasc Access 2006;7:53-59.

3. Jadoul M, Thumma J, Fuller DS, et al. Modifiable practices associated with sudden death among hemodialysis patients in the Dialysis Outcomes and Practice Patterns Study. Clin J Am Soc Nephrol 2012;7:765-774.

4. Singh S, Choi P, Power A, et al. Ten-year patient survival on maintenance haemodialysis: association with treatment time and dialysis dose. J Nephrol 2013;26:763-770.

5. Hassell DR, van der Sande FM, Kooman JP, Tordoir JP, Leunissen KM. Optimizing dialysis dose by increasing blood flow rate in patients with reduced vascular-access flow rate. Am J Kidney Dis 2001;38:948-955.

6. Kimata N, Karaboyas A, Bieber BA, et al. Gender, low Kt/V, and mortality in Japanese hemodialysis patients: opportunities for improvement through modifiable practices. Hemodial Int 2014;18:596-606.

7. Tentori F, Zhang J, Li Y, et al. Longer dialysis session length is associated with better intermediate outcomes and survival among patients on in-center three times per week hemodialysis: results from the Dialysis Outcomes and Practice Patterns Study (DOPPS). Nephrol Dial Transplant 2012;27:4180-4188.

8. Pisoni RL, Arrington CJ, Albert JM, et al. Facility hemodialysis vascular access use and mortality in countries participating in DOPPS: an instrumental variable analysis. Am J Kidney Dis 2009;53:475-491. 
9. Arbor Research Collaborative for Health. DOPPS practice monitor [Internet]. Ann Arbor (MI): Arbor Research Collaborative for Health, c2015 [cited 2015 Dec 16]. Available from: http://www.dopps.org/DPM/Default.aspx.

10. Bloembergen WE, Stannard DC, Port FK, et al. Relationship of dose of hemodialysis and cause-specific mortality. Kidney Int 1996;50:557-565.

11. Borzou SR, Gholyaf M, Zandiha M, Amini R, Goodarzi MT, Torkaman B. The effect of increasing blood flow rate on dialysis adequacy in hemodialysis patients. Saudi J Kidney Dis Transpl 2009;20:639-642.

12. Kim YO, Song WJ, Yoon SA, et al. The effect of increasing blood flow rate on dialysis adequacy in hemodialysis patients with low Kt/V. Hemodial Int 2004;8:85.

13. Eknoyan G, Beck GJ, Cheung AK, et al. Effect of dialysis dose and membrane flux in maintenance hemodialysis. N Engl J Med 2002;347:2010-2019.

14. Gutzwiller JP, Schneditz D, Huber AR, Schindler C, Garbani E, Zehnder CE. Increasing blood flow increases kt/ $\mathrm{V}$ (urea) and potassium removal but fails to improve phosphate removal. Clin Nephrol 2003:59:130-136.

15. Vanholder R, Glorieux G, Eloot S. Once upon a time in dialysis: the last days of Kt/V? Kidney Int 2015;88:460-465. 